\title{
Statin-induced anti-HMGCR myopathy: successful therapeutic strategies for corticosteroid-free remission in 55 patients
}

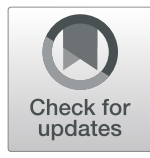

\author{
Alain Meyer ${ }^{1}$, Yves Troyanov ${ }^{2,3}$, Julie Drouin ${ }^{2,4}$, Geneviève Oligny-Longpréé, ${ }^{2,5}$, Océane Landon-Cardinal2, ${ }^{2,5}$, \\ Sabrina Hoa ${ }^{2,5,6}$, Baptiste Hervier ${ }^{7}$, Josiane Bourré-Tessier ${ }^{2,5,6}$, Anne-Marie Mansour ${ }^{2,8}$, Sara Hussein ${ }^{2,5}$, \\ Vincent Morin ${ }^{9}$, Eric Rich 2,5,6, Jean-Richard Goulet ${ }^{2,5}$, Sandra Chartrand ${ }^{2,10}$, Marie Hudson 11,12,13, Jessica Nehme ${ }^{2,8}$, \\ Jean-Paul Makhzoum ${ }^{2,8}$, Farah Zarka ${ }^{2,8}$, Edith Villeneuve ${ }^{2,5}$, Jean-Pierre Raynauld ${ }^{2,5}$, Marianne Landry ${ }^{2,8}$, \\ Erin K. O'Ferrall ${ }^{14,15,19}$, Jose Ferreira ${ }^{16,17}$, Benjamin Ellezam ${ }^{16,18}$, Jason Karamchandani ${ }^{15,19}$, Sandrine Larue ${ }^{20,21}$, \\ Rami Massie ${ }^{14,15}$, Catherine Isabelle 20,22 , Isabelle Deschênes ${ }^{20,23}$, Valérie Leclair ${ }^{11,12}$, Hélène Couture ${ }^{24,25}$, \\ Ira N. Targoff ${ }^{26,27}$, Marvin J. Fritzler ${ }^{28}$ and Jean-Luc Senécal ${ }^{2,5,6^{*}}$
}

\begin{abstract}
Objective: To describe successful therapeutic strategies in statin-induced anti-HMGCR myopathy. Methods: Retrospective data from a cohort of 55 patients with statin-induced anti-HMGCR myopathy, sequentially stratified by the presence of proximal weakness, early remission, and corticosteroid and IVIG use at treatment induction, were analyzed for optimal successful induction and maintenance of remission strategies.

Results: A total of 14 patients achieved remission with a corticosteroid-free induction strategy (25\%). In 41 patients treated with corticosteroids, only 4 patients (10\%) failed an initial triple steroid/IVIG/steroid-sparing immunosuppressant (SSI) induction strategy. Delay in treatment initiation was independently associated with lower odds of successful maintenance with immunosuppressant monotherapy (OR 0.92, 95\% Cl 0.85 to $0.97, P=0.015)$. While 22 patients (40\%) presented with normal strength, only 9 had normal strength at initiation of treatment.

Conclusion: While corticosteroid-free treatment of anti-HMGCR myopathy is now a safe option in selected cases, initial triple steroid/IVIG/SSI was very efficacious in induction. Delays in treatment initiation and, as a corollary, delays in achieving remission decrease the odds of achieving successful maintenance with an SSI alone. Avoiding such delays, most notably in patients with normal strength, may reset the natural history of anti-HMGCR myopathy from a refractory entity to a treatable disease.
\end{abstract}

Keywords: Autoimmune myositis, Immune-mediated necrotizing myopathy, Anti-HMGCR myopathy, Statin, Therapy, Corticosteroid-free therapy, Immunosuppressant, IVIG, Remission

\footnotetext{
* Correspondence: dr.j.l.senecal.md@gmail.com

${ }^{2}$ Department of Medicine, Faculty of Medicine, University of Montreal, Montreal, Québec, Canada

${ }^{5}$ Division of Rheumatology, Centre Hospitalier de I'Université de Montréal (CHUM), 264, Boulevard René-Lévesque Est, Montréal, Québec H2X 1P1,

Canada

Full list of author information is available at the end of the article
}

(c) The Author(s). 2020 Open Access This article is distributed under the terms of the Creative Commons Attribution 4.0 International License (http://creativecommons.org/licenses/by/4.0/), which permits unrestricted use, distribution, and reproduction in any medium, provided you give appropriate credit to the original author(s) and the source, provide a link to the Creative Commons license, and indicate if changes were made. The Creative Commons Public Domain Dedication waiver (http://creativecommons.org/publicdomain/zero/1.0/) applies to the data made available in this article, unless otherwise stated. 


\section{Rheumatology key messages}

- Anti-HMGCR myopathy with normal strength is common, and a corticosteroid-free induction strategy should be considered.

- In patients with proximal weakness, induction with corticosteroids + IVIG + a corticosteroid-sparing immunosuppressant is efficacious and may allow accelerated corticosteroid tapers.

- Targeting early remission increases the efficacy of a corticosteroid-sparing immunosuppressant regimen in maintaining remission.

\section{Introduction}

Statin-induced immune-mediated necrotizing myopathy (IMNM) was initially described in patients on statin therapy who, despite statin discontinuation, developed a persistent myopathy, responsive only to immunosuppression [1]. It was later found that autoantibodies to 3-hydroxy-3-methyl-glutaryl-coenzyme A reductase (HMGCR) define that myopathy [2-4], that very high serum levels of creatine kinase (CK) and widespread damage on magnetic resonance imaging (MRI) are common [5], that sarcolemmal and capillary membrane attack complex (MAC) deposition are present on muscle biopsy [2, 6-8], and that intense immunosuppressive treatment is often needed [2, 7-12]. Recently, the pathogenicity of anti-HMGCR was demonstrated [13-15], and during the 224th European Neuromuscular Centre (ENMC) International Workshop [16], in the presence of proximal weakness and elevated CK levels, anti-HMGCR myopathy was defined. Compellingly, limb-girdle muscular dystrophy presentation [17] and isolated hyperCKemia [8] were reported as part of the spectrum of anti-HMGCR myopathy.

Therapeutically, efficacy of intravenous immunoglobulin (IVIG) monotherapy in statin-induced anti-HMGCR myopathy introduced the concept of corticosteroid-free induction strategy [18]. In parallel, a corticosteroid-based induction strategy composed of corticosteroids, IVIG, and a steroidsparing immunosuppressant (SSI) was proposed as the initial treatment of severe anti-HMGCR myopathy [10]. Since this disease occurs in older patients who often have diabetes mellitus [19] and cardiovascular disease, corticosteroid-free induction and maintenance strategies are of utmost interest to minimize treatment-related morbidity $[20,21]$.

From a cohort of 55 patients, we studied the natural history and the spectrum of severity of untreated and treated statininduced anti-HMGCR myopathy, while examining the therapeutic strategies that ultimately led to steroid-free remission.

\section{Methods}

\section{Patients}

The PHESEMO study (PHEnotype, SErology, and successful MOnotherapy maintenance in Autoimmune Myositis) is a retrospective study of patients with autoimmune myositis
(AIM) followed longitudinally at Centre Hospitalier de l'Université de Montréal (CHUM) and Hôpital du Sacré-Coeur de Montréal (Montreal, QC, Canada) from 2001 to 2018. For the STATIN-PHESEMO study, only patients with statin-induced anti-HMGCR myopathy were considered, and additional patients from two University of Montreal affiliated hospitals (Centre Hospitalier Affilié Universitaire Régional de TroisRivières and Hôpital Maisonneuve-Rosemont) were included. The STATIN-PHESEMO study was approved by the CHUM Research Ethics Committee (reference number 2015-5607CE14.248) and by the Research Ethics Committees of Hôpital du Sacré-Coeur (2014-1042), Centre Hospitalier Affilié Universitaire Régional de Trois-Rivières (2014-028-03), and Hôpital Maisonneuve-Rosemont (2015-639-CER14107).

The terminology of anti-HMGCR myopathy in this study refers only to patients with a statin-induced anti-HMGCR myopathy. Definite anti-HMGCR myopathy is defined as positive anti-HMGCR autoantibodies, elevated serum CK levels, and proximal skeletal muscle weakness (16). Probable anti-HMGCR myopathy was defined for this study as positive antiHMGCR, elevated CK levels, suggestive muscle biopsy findings with necrosis/regeneration or MAC deposition, and normal strength. Possible anti-HMGCR myopathy was defined for this study as positive antiHMGCR, elevated CK levels, and normal strength, irrespective of normal or absent muscle biopsy results.

\section{Data collection}

Data on history, physical findings, and investigations were collected by retrospective medical record review using a standardized protocol. Data collection focused on demographics, myopathic features, chronology of events leading to the diagnosis (statin use, CKs, and clinical manifestations), treatment strategies (induction vs maintenance), and muscle biopsy findings.

\section{Definitions for therapy, remission, maintenance, and severity}

These are shown in Additional file 1: Table S1.

\section{Identifying therapeutic subgroups within the STATIN- PHESEMO study}

These are shown in Additional file 2: Table S2.

\section{Serology}

Autoantibodies to HMGCR were detected by an addressable laser bead immunoassay (ALBIA) using a laboratory developed test (Mitogen Advanced Diagnostics, Calgary, AB, Canada) that was validated and then replaced by a commercially available ELISA (Inova Diagnostics, San Diego, CA, USA). Other AIM autoantibodies were detected by a 
commercial line immunoassay (Euroimmun $\mathrm{GmbH}$, Lübeck, Germany) and included those directed to Jo-1, Mi2- $\alpha$, Mi2- $\beta$, MDA5, NXP2, TIF1 $\gamma$, PL7, PL12, PM/Scl75, PM/Scl100, Ku, SRP, EJ, OJ, and Ro52/TRIM21 autoantigens.

\section{Statistical analysis}

Descriptive statistics were used to summarize the baseline characteristics of the study cohort. Continuous data were reported as medians with ranges, and categorical data were presented as counts with percentages.

To identify predictors of successful maintenance of remission with SSI monotherapy, we first used univariate logistic regression models to quantify the association between monotherapy maintenance and age, sex, CK, presence of normal strength and dysphagia at treatment initiation, and delay in treatment initiation, as well as use of corticosteroids and IVIG in induction. Then, a multivariate logistic regression model was done to identify independent predictors of monotherapy maintenance, incorporating variables that were significantly associated with monotherapy maintenance in univariate analyses. To account for potential residual confounding, we performed sensitivity analyses which additionally adjusted for omitted variables.

\section{Results}

\section{Clinical characteristics of 55 patients with anti-HMGCR myopathy}

Table 1 details baseline characteristics: the median age at diagnosis was 67.7 years, 95\% were Caucasians, $72 \%$ had diabetes mellitus, and none had cancer within 3 years of diagnosis. The statin most commonly prescribed was atorvastatin (84\%). A total of 22 patients (40\%) presented with normal strength and elevated CK levels. Statin was discontinued in every patient.

At treatment initiation, 46 patients $(84 \%)$ had proximal weakness, the median CK elevation was $5000 \mathrm{UI} / \mathrm{L}$ (range 554-23,000), 48 patients had biopsy evidence of a necrotizing myopathy, and all were positive for antiHMGCR autoantibodies. Eighty-four percent (46/55) of patients had definite, $13 \%$ (7/55) probable, and 3\% (2/55) possible anti-HMGCR myopathy.

\section{Corticosteroid-free induction strategies were successful in all 14 selected patients}

The chronology of events leading to the initiation of treatment is detailed in Additional file 3: Table S3. Extensive delay between presentation and treatment was seen in 2 patients (57 and 78 months); interestingly, on statin discontinuation, CK levels had fallen under $500 \mathrm{UI} / \mathrm{L}$, but ultimately rose to $>2100 \mathrm{U} / \mathrm{L}$, leading to treatment.
Table 1 Baseline characteristics of patients with anti-HMGCR myopathy $(N=55)$

\begin{tabular}{|c|c|}
\hline & $\begin{array}{l}n(\%) \text { or median } \\
\text { (range) }\end{array}$ \\
\hline Sex, male/female & $30 / 25$ \\
\hline Age at diagnosis, median (range), years & $67.7(44-86.1)$ \\
\hline Prior statin use, $n(\%)$ & $55(100)$ \\
\hline Atorvastatin use, $n(\%)$ & $46(84)$ \\
\hline Diabetes mellitus, $n(\%)$ & $39(72)$ \\
\hline Cardiovascular disease, $n(\%)^{*}$ & $29(53)$ \\
\hline Cancer within 3 years of diagnosis, $n$ & 0 \\
\hline $\begin{array}{l}\text { First serum CK levels at presentation, } \\
\text { median (range), UI/L }\end{array}$ & $2935(500-19,465)$ \\
\hline $\begin{array}{l}\text { CK levels at treatment initiation, } \\
\text { median (range), UI/L }\end{array}$ & $5000(554-23,000)$ \\
\hline Myalgias, $n(\%)$ & $21(38)$ \\
\hline Subjective oropharyngeal dysphagia, $n(\%)$ & $16(29)$ \\
\hline Objective oropharyngeal dysphagia, $n$ (\%) & $5(9)$ \\
\hline $\begin{array}{l}\text { Proximal muscle weakness at } \\
\text { presentation, } n(\%)\end{array}$ & $33(60)$ \\
\hline $\begin{array}{l}\text { Proximal muscle weakness at } \\
\text { treatment initiation, } n(\%)\end{array}$ & $46(84)$ \\
\hline Muscle biopsy, n (\%) & $54(98)$ \\
\hline Necrosis and regeneration, $n(\%)$ & $48(87)$ \\
\hline $\begin{array}{l}\text { Isolated sarcolemmal/capillary } \\
\text { MAC deposition, } n(\%)\end{array}$ & $4(7)$ \\
\hline Regeneration only, $n$ (\%) & $1(2)$ \\
\hline Normal, $n(\%)$ & $1(1)$ \\
\hline \multicolumn{2}{|l|}{ Other abnormalities } \\
\hline $\begin{array}{l}\text { MHC-1 expression on non-necrotic } \\
\text { muscle fibers, } n(\%)\end{array}$ & $26 / 51(51)$ \\
\hline $\begin{array}{l}\text { Sarcolemmal and/or capillary } \\
\text { MAC deposition, } n(\%)^{* *}\end{array}$ & $38 / 42(90)$ \\
\hline
\end{tabular}

*Myocardial infarction or stroke

**MAC deposition was found on non-necrotic fibers and/or endomysial capillaries

As shown in Fig. 1, the corticosteroid-free cohort consisted of 14 patients with a successful induction. Initial induction strategies were SSI monotherapy $(n=7)$ and Dual IVIG/SSI monotherapy $(n=7)$. As illustrated in Additional file 4: Table S4, the successful induction strategies were as follows: SSI monotherapy ( $n=6$ patients), SSI combination $(n=1)$, Dual IVIG/SSI monotherapy $(n=4)$, and Dual IVIG/SSI combination $(n=3)$. All evaluable maintenance strategies $(n=12)$ were successful.

The Solo SSI cohort included 7 patients with a median CK level of $1720 \mathrm{UI} / \mathrm{L}$ (range 554-3257), 3 (43\%) of whom had proximal weakness but none considered severe (Fig. 1). As seen in Additional file 4: Table S4, induction with MTX alone was successful in 6 patients, with time to remission ranging from 1.9 to 34 months. An AZA/MTX step-up strategy 


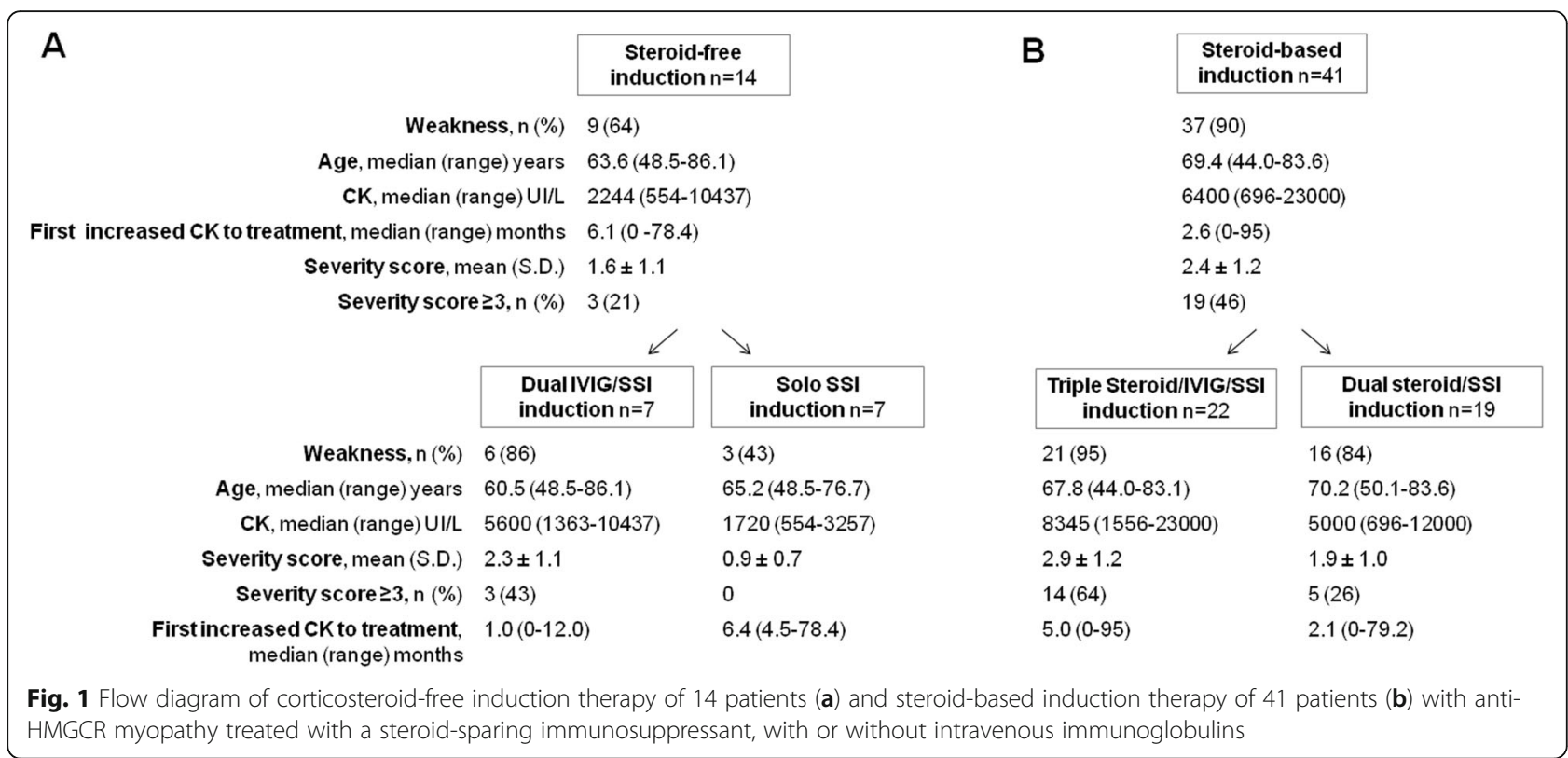

was successful in 1 patient (no. 6) who had failed initial AZA induction monotherapy.

The Dual IVIG/SSI cohort included 7 patients with a median CK level of $5600 \mathrm{UI} / \mathrm{L}$ (range 1363-10,437), 6 (86\%) of whom had proximal weakness and 3 (43\%) with severe myopathy (Fig. 1). Initial induction with MTX/ IVIG was successful in 4 patients, with time to remission ranging from 1 to 5 months. For the remaining 3 patients, successful step-up induction strategies of MTX/ AZA/IVIG were needed.

\section{Corticosteroid-based induction strategies were successful in 41 patients}

As shown in Fig. 1b and Table 2, the corticosteroid-based induction cohort consisted of 41 patients. Initial induction strategies were Dual steroid/SSI monotherapy $(n=19)$ and Triple steroid/IVIG/SSI monotherapy $(n=22)$. Adequate induction corticosteroid therapy was given in 36 (88\%) patients. The decision to include IVIG in the induction strategy was left to the treating physician and was determined by the perceived severity of disease (confounded by indication) or failure of a Dual steroid/SSI induction strategy ( $n=3$ patients). Proximal weakness was present in 37 (90\%) patients, and 19 (46\%) had severe myopathy. Serum CK elevation ranged broadly from 696 to 23,000 UI/L. Delay before treatment initiation was also wide, ranging from immediate treatment to 95 months.

All corticosteroid-based induction strategies $(n=41)$ were successful. As seen in Fig. 1 and Table 2, patients were stratified first for initial IVIG use and then for early or late remission. Overall, only $52.6 \%(n=10)$ of patients in the Dual steroid/SSI cohort and $54.5 \%(n=12)$ of patients in the Triple steroid/IVIG/SSI cohort had an early remission.
Importantly, patients with an early remission had a shorter median delay from presentation to treatment compared to patients with late remission (1.07 vs 12 months, $P=0.043)$.

Patients with a late remission $(n=9)$ in the Dual steroid/SSI cohort were analyzed (Table 2 and data not shown). Adequate induction corticosteroid therapy was given in all but 2 patients. Optimizing SSI therapy to achieve remission was frequent, as efficacious doses of MTX were 20-30 mg/week ( $n=7)$, MMF 3 g/day $(n=1)$, and AZA/ALLO $(n=1)$. The initial induction strategy was successful in 8 patients, with a median time to remission of 7 months (range 4-22 months). An AZA/ALLO switching induction strategy was successful in 1 patient who had failed initial steroid/MTX induction therapy.

Patients with a late remission $(n=10)$ in the Triple steroid/IVIG/SSI cohort were also analyzed. Adequate induction corticosteroid therapy was given in 8 patients. For 3 patients with successful initial induction strategy, time to remission was between 4 and 5 months. For 3 additional patients, initial induction strategy was successful only when IVIG therapy was added. Time to remission of the latter patients was 13-18 months, but the late addition of IVIG therapy resulted in remission in $\leq$ 3 months. Failure of a Triple steroid/IVIG/SSI induction strategy was seen in the last 4 patients, and they had by definition refractory anti-HMGCR myopathy.

Induction strategies used in refractory anti-HMGCR $(n=4)$ were switching $(n=1)$ or step-up $(n=3)$. One patient achieved remission with an AZA/ALLO switching induction strategy, while 3 patients had successful step-up induction strategy with MTX/AZA, MTX/RTX, and MMF/ABA, respectively. For the 4 refractory patients, time to remission from the initial induction strategy was $8,18,18$, and 53 months, respectively. 
Table 2 Severity factors in patients with anti-HMGCR myopathy and successful steroid-based induction therapy, stratified by concomitant use of IVIG therapy and early vs late remission $(N=41)$

\begin{tabular}{|c|c|c|c|c|}
\hline & \multicolumn{2}{|c|}{ Dual steroids/SSI cohort $(n=19)$} & \multicolumn{2}{|c|}{ Triple steroids/IVIG/SSI cohort $(n=22)$} \\
\hline & $\begin{array}{l}\text { Early remission }(\leq 3 \\
\text { months), } n=10\end{array}$ & $\begin{array}{l}\text { Late remission ( }>3 \\
\text { months), } n=9\end{array}$ & $\begin{array}{l}\text { Early remission }(\leq 3 \\
\text { months), } n=12\end{array}$ & $\begin{array}{l}\text { Late remission (>3 } \\
\text { months), } n=10\end{array}$ \\
\hline Weakness at treatment onset, $n(\%)$ & $8(80)$ & $8(89)$ & $11(92)$ & $10(100)$ \\
\hline Age at treatment onset, median (range) years & $70.6(59.9-83.6)$ & $69.4(50.1-81.3)$ & $73.6(46.5-83.1)$ & $60.4(44.0-74.3)$ \\
\hline CK level at treatment onset, median (range) UI/L & $2673(696-12,000)$ & $6405(3573-10,465)$ & $7317(1556-13,339)$ & $10,789(2267-23,000)$ \\
\hline Severity score, mean (SD) & $1.4(0.8)$ & $2.5(0.9)$ & $2.5(1.2)$ & $3.3(0.9)$ \\
\hline Severity score $\geq 3, n(\%)$ & $1(10)$ & $4(44)$ & $6(50)$ & $8(80)$ \\
\hline $\begin{array}{l}\text { Delay from first increased serum CK (> } 500 \mathrm{UI} / \mathrm{L}) \text { to } \\
\text { treatment, median (range) months }\end{array}$ & $1.4(0-79.2)$ & $13.4(0-24.9)$ & $0.8(0-42.2)$ & $11.5(0-95)$ \\
\hline $\begin{array}{l}\text { Delay from treatment to serum } C K<500 \mathrm{UI} / \mathrm{L} \text {, median } \\
\text { (range) months }\end{array}$ & $1.7(0.4-3)$ & $11.5(4-50.7)$ & $2.0(0.6-3)$ & $15.0(3.2-53)$ \\
\hline \multicolumn{5}{|l|}{ Needed induction strategies to obtain remission, $n(\%)$} \\
\hline 1 & $10(100)$ & $8(89)$ & $12(100)$ & $6(60)$ \\
\hline 2 & 0 & $1(11)$ & 0 & $1(10)$ \\
\hline 3 & 0 & 0 & 0 & $2(20)$ \\
\hline$\geq 4$ & 0 & 0 & 0 & $1(10)$ \\
\hline Successful maintenance with SSI monotherapy, $n$ (\%) & $8(80)$ & $8(89)$ & $5(42)$ & $2(20)$ \\
\hline \multicolumn{5}{|l|}{ Corticosteroid dosage at last follow-up } \\
\hline No corticosteroids & $8(80)$ & $8(89)$ & $9(75)$ & $8(80)$ \\
\hline Prednisone $\leq 5 \mathrm{mg}$ per day & $2(20)$ & $1(11)$ & $1(8)$ & $1(10)$ \\
\hline Prednisone $>5 \mathrm{mg}$ per day & 0 & 0 & $2(17)$ & $1(10)$ \\
\hline Drug-free remission, $n(\%)$ & $3(30)$ & $1(11)$ & 0 & 0 \\
\hline Normal strength at last follow-up, $n(\%)$ & $9(90)$ & $8(89)$ & $6(50)$ & $5(50)$ \\
\hline
\end{tabular}

SSI steroid-sparing immunosuppressant

\section{Corticosteroid-free maintenance was successful in $73 \%$ of patients treated at induction with corticosteroids}

As shown in Table 3, a corticosteroid-free SSI monotherapy maintenance strategy was successful in 22 patients, while a SSI monotherapy maintenance with daily prednisone $\leq 5 \mathrm{mg}$ was effective in one patient. Normal strength at last follow-up was seen in $87 \%(n=20 / 23)$ of patients. Drug-free remission was possible in 4 patients.

In the remaining 18 patients not meeting the definition of successful maintenance strategy with SSI monotherapy, 8 patients had a steroid-free successful maintenance strategy: 4 patients had a maintenance strategy of SSI monotherapy plus IVIG whereas 4 patients had a maintenance strategy of an SSI combination (with or without IVIG) (Table 3). Overall, $73 \%(n=30 / 41)$ of patients treated with corticosteroids at induction had a successful steroid-free maintenance.

In this corticosteroid-based induction cohort, the SSIs used for successful maintenance, alone or in combination, were $\operatorname{MTX}(n=25)$, AZA $(n=3)$, AZA/ALLO $(n=2)$, $\operatorname{MMF}(n=7)$, and ABA $(n=1)$. SSI combinations used successfully (with or without IVIG) were MTX/AZA ( $n=$ $2)$, MTX/MMF $(n=2)$, and MMF/ABA $(n=1)$. The median follow-up was 52 months (range 14-140 months).
Early diagnosis of anti-HMGCR myopathy with hyperCKemia but normal strength favored corticosteroidfree induction strategies

As shown in Fig. 2, 22/55 (40\%) patients had no weakness at presentation and initiation of treatment, and hyperCKemia was the first manifestation of anti-HMGCR myopathy. The median CK level at presentation of these 22 patients was 1509 UI/L (range 500-5613 UI/L).

Figure 2 also shows that corticosteroid-free induction was used in $56 \%(n=5 / 9)$ in the early treatment cohort, versus $8 \%$ $(n=1 / 13)$ in the delayed treatment cohort $(P=0.023)$, indicating that patients with anti-HMGCR myopathy with normal strength were candidates for corticosteroid-free induction.

The chronology of events leading to treatment in these 22 patients is detailed in Additional file 5: Table S5. Interestingly, after statin discontinuation, serum CK levels dropped by $\geq 50 \%$ in 6 (27\%) of these 22 patients.

\section{Early treatment of anti-HMGCR myopathy increased the efficacy of SSI monotherapy in maintaining remission} The first evidence of a long-term benefit of treating antiHMGCR myopathy early, i.e., successful corticosteroid-free SSI monotherapy maintenance, is illustrated in Table 3 . The 
Table 3 Severity factors for successful steroid-free maintenance therapy in patients with anti-HMGCR myopathy stratified by the use of steroid-sparing immunosuppressants in monotherapy or in combination, with or without IVIG $(N=41)$

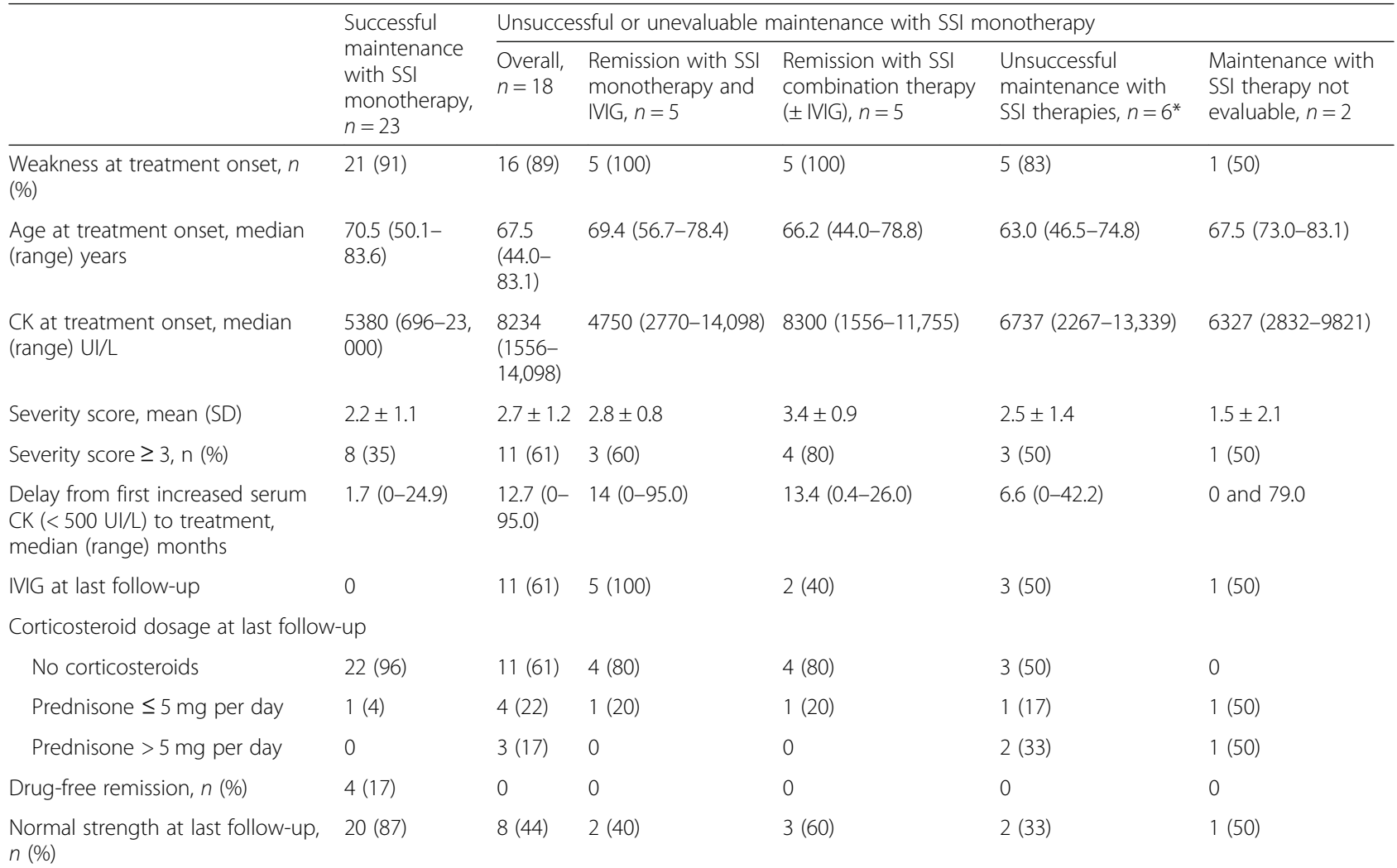

SSI steroid-sparing immunosuppressant

*Unsuccessful maintenance with SSI therapy included failure to SSI monotherapy $(n=3)$, SSI monotherapy + IVIG $(n=2)$ and SSI combination therapy + IVIG $(n=1)$

median delays in initiating treatment in patients with successful SSI monotherapy maintenance $(n=23)$ vs those without $(n=18)$ were 1.7 month and 12.7 months, respectively $(P=0.048)$, in favor of early intervention.

The second evidence is shown in Fig. 2. The rate of successful remission maintenance with SSI monotherapy was $64 \%$ in the immediate treatment cohort as opposed to only $31 \%(P=0.056)$ in the delayed treatment cohort (median delay to treatment 0.75 vs 21.6 months, respectively).

Table 4 presents statistical analyses of predictive factors for successful SSI monotherapy maintenance. Delay in treatment initiation was independently associated with a lower odds of successful maintenance with an SSI monotherapy (OR 0.92, $95 \%$ CI 0.85 to $0.97, P=0.015$ ). In addition, IVIG use in induction was strongly and significantly associated with a reduced odds of successful monotherapy maintenance (OR $0.08,95 \%$ CI 0.01 to $0.32, P=0.001$ ). Sensitivity analyses additionally adjusted for age, sex, strength, CK, dysphagia, and corticosteroid use were consistent with these results.

\section{Discussion}

This case series provides an overview of the disease spectrum of statin-induced anti-HMGCR myopathy, ranging from presentation as an acute IMNM [2] to persistent hyperCKemia despite statin discontinuation. The initial 12 patients from the present cohort were described previously [8], and thereon, access to anti-HMGCR autoantibody testing allowed diagnosis of anti-HMGCR myopathy in 43 additional patients.

The initial description of 8 patients with a progressive, MHC-I positive myopathy associated with statin therapy was noteworthy for their complete response to MTX and prednisone [1]. Subsequent reports demonstrated that anti-HMGCR myopathy was difficult to treat [7-10] and that younger patients were harder to treat than older patients [11].

There is no uniform approach to the treatment of antiHMGCR myopathy [16, 22-24], nor are there a described severity score [2] or treat to target recommendations [25]. The 224th ENMC definition of severe anti-HMGCR myopathy was the presence of walking difficulties and/or dysphagia, while partial remission was defined as an improvement $\geq$ $110 \%$ of MMT-8 and/or CK levels, the latter remaining greater than or equal to twice the normal range, i.e., $\geq 500 \mathrm{UI} /$ $\mathrm{L}$ [16]. The definition of complete remission consisted of normal strength and normal serum CK levels [16].

Achieving sustained remission with normal CK levels, normal strength, and no corticosteroids is indeed a goal 


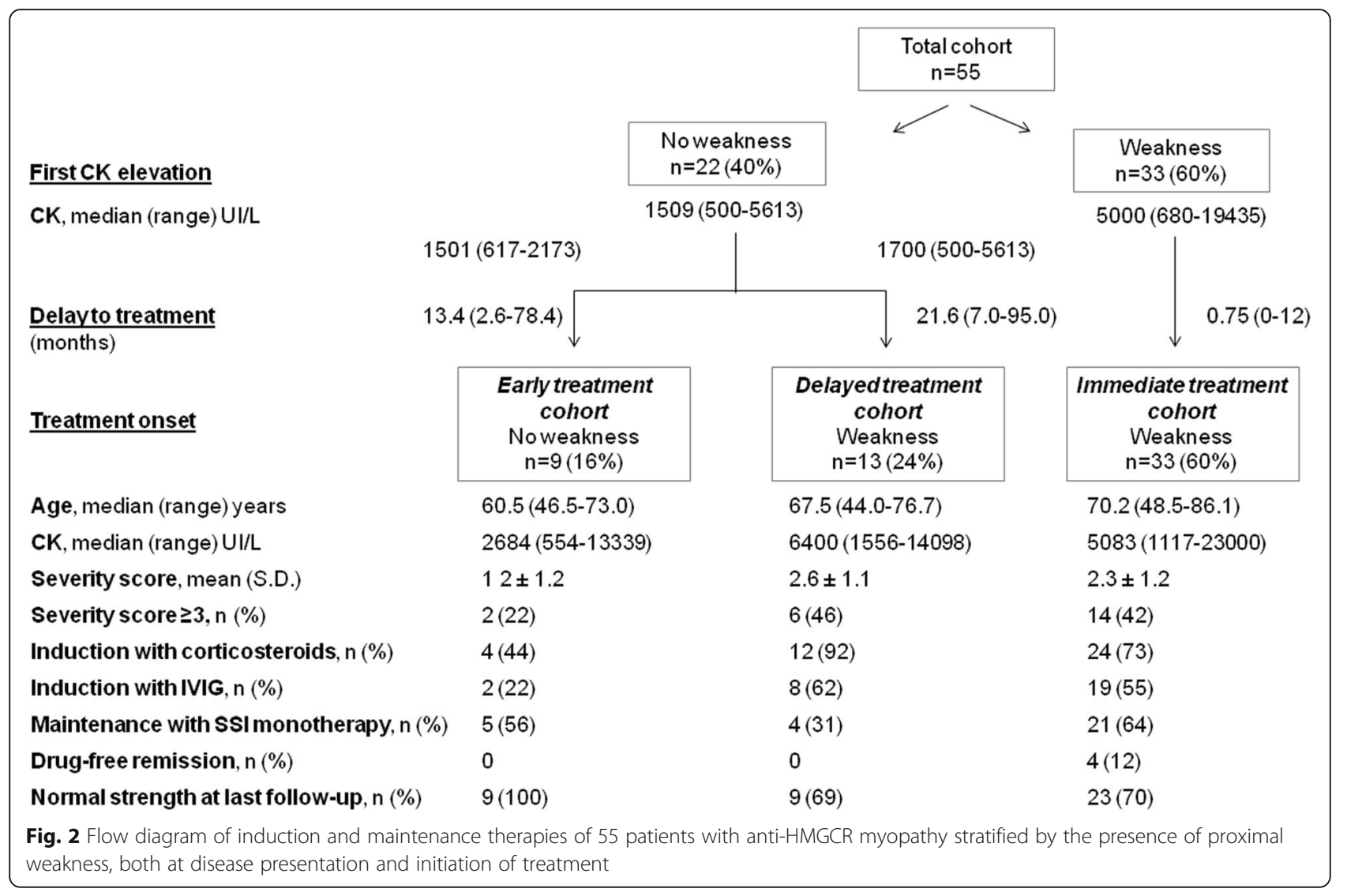

Table 4 Univariate and multivariate analyses of predictive factors for successful maintenance with steroid-sparing immunosuppressant monotherapy in patients with anti-HMGCR myopathy $(N=55)$

\begin{tabular}{|c|c|c|c|c|}
\hline & \multicolumn{2}{|c|}{ Maintenance of remission with SSI monotherapy } & \multirow{2}{*}{$\begin{array}{l}\text { Univariate } \\
\text { OR }(95 \% \mathrm{Cl}), P \\
\text { value }\end{array}$} & \multirow{2}{*}{$\begin{array}{l}\text { Multivariate } \\
\text { OR }(95 \% \text { Cl), P } \\
\text { value }\end{array}$} \\
\hline & $\begin{array}{l}\text { Yes }(n=30), n(\%) \text { or } \\
\text { Median (range) }\end{array}$ & $\begin{array}{l}\text { No }(n=25), n(\%) \text { or } \\
\text { Median (range) }\end{array}$ & & \\
\hline Age at treatment initiation, Median (range) years & $68.2(48.5-86.1)$ & $66.9(44.0-83.1)$ & $\begin{array}{l}1.02(0.97 \text { to } 1.08) \\
P=0.516\end{array}$ & - \\
\hline Male sex, $n(\%)$ & $17(57)$ & $13(52)$ & $\begin{array}{l}1.21(0.41 \text { to } 3.54) \\
P=0.729\end{array}$ & - \\
\hline Normal strength at treatment initiation, $n(\%)$ & $5(17)$ & $4(16)$ & $\begin{array}{l}1.05(0.25 \text { to } 4.72) \\
P=0.947\end{array}$ & - \\
\hline CK at treatment initiation, Median (range) $\mathrm{UI} / \mathrm{L}$ & $4959(554-23,000)$ & $5083(1533-14,098)$ & $\begin{array}{l}1.00(1.00 \text { to } 1.00) \\
P=0.582\end{array}$ & - \\
\hline Dysphagia, subjective, $n$ (\%) & $9(30)$ & $7(28)$ & $\begin{array}{l}1.10(0.34 \text { to } 3.65) \\
P=0.871\end{array}$ & - \\
\hline Dysphagia, objective, $n(\%)$ & $1(3)$ & $4(16)$ & $\begin{array}{l}0.18(0.01 \text { to } 1.33) \\
P=0.139\end{array}$ & - \\
\hline $\begin{array}{l}\text { Delay between first increased serum CK and treatment } \\
\text { initiation, Median (range) months }\end{array}$ & $2.0(0-24.9)$ & $11.0(0-95.0)$ & $\begin{array}{l}0.94(0.88 \text { to } 0.98) \\
P=0.025\end{array}$ & $\begin{array}{l}0.92(0.85 \text { to } 0.97), \\
P=0.015\end{array}$ \\
\hline Use of corticosteroids in induction, $n(\%)$ & $23(77)$ & $18(72)$ & $\begin{array}{l}1.28(0.37 \text { to } 4.39) \\
P=0.693\end{array}$ & - \\
\hline Use of IVIG in induction, $n(\%)$ & $10(33)$ & $19(76)$ & $\begin{array}{l}0.16(0.04 \text { to } 0.50) \\
P=0.002\end{array}$ & $\begin{array}{l}0.08 \text { (0.01 to } 0.32) \\
P=0.001\end{array}$ \\
\hline
\end{tabular}


of treatment. But both steroid myopathy and MRIdocumented damage may occur [4], and remission may be present without full recovery of strength. In a large cohort of treated anti-HMGCR myopathy, strength recovery was often seen with persistent serum CK elevation $>500 \mathrm{UI} / \mathrm{L}$, a sign of ongoing activity [11]. In another study, CK levels were shown to be closely associated with disease activity [25]. In the present cohort of 55 patients, hyperCKemia $\geq 500 \mathrm{UI} / \mathrm{L}$ with normal strength was the presentation of $40 \%$ of patients. Taken altogether, these results suggest that in anti-HMGCR myopathy, achieving a serum CK level $\leq 500 \mathrm{UI} / \mathrm{L}$ could define remission and be the goal of both successful induction and maintenance strategies. Targeting early remission may be justified to minimize steroid therapy [21]. Indeed, IVIG use in the 3 months after presentation of necrotizing myopathy was associated with better outcomes at 6 months [10].

In the present study, analysis of the corticosteroid-free induction cohort highlighted the relative contributions of IVIG and an SSI in an induction strategy. Patients with successful Solo SSI strategy achieved remission up to 13 months after initiation of treatment. In contrast, patients with a successful Dual IVIG/SSI strategy often achieved remission within 3 months, illustrating the efficacy of IVIG. Steroid-free induction strategies were demonstrated to be efficacious, thus sparing steroid toxicity in an older population with diabetes and cardiovascular disease. As shown by Mammen and Tiniakou, further studies will identify ideal candidates for steroidfree induction of anti-HMGCR myopathy [18].

Analysis of the corticosteroid-based induction cohort confirmed that a Triple IVIG/steroid/SSI induction was efficacious in most patients since only $4 / 22$ (18\%) patients failed that induction strategy. As for treating antiHMGCR myopathy with a Dual steroid/SSI strategy, late remissions were frequent, inferring that early optimization of SSI is essential and corticosteroids less efficacious than previously thought. The 224th ENMC recommended prednisone $1 \mathrm{mg} / \mathrm{kg} /$ day [16] for treatment of severe antiHMGCR myopathy. However, tapering corticosteroids only when CK levels have normalized, as recommended in general for AIM treatment [26, 27], may not apply to antiHMGCR myopathy. Indeed, if IVIG and an optimized SSI are introduced as the initial treatment, it is possible to tailor initial steroid dosing to both comorbidities and disease severity, and the promptness of the corticosteroid taper to the early CK response, therefore tapering corticosteroids even when CK levels have not yet normalized.

In this study, the corticosteroid-based induction strategy achieved steroid-free remission in $73 \%$, normal strength at last follow-up in $68 \%$, and steroid-free successful maintenance with SSI monotherapy in 54\% of patients. In contrast, the corticosteroid-free induction strategy achieved steroidfree remission in $100 \%$, normal strength at last follow-up in
93\%, and successful maintenance with an SSI monotherapy in $50 \%$ of patients. Successful, steroid-free maintenance with either SSI monotherapy with IVIG, or combination SSI therapy (with or without IVIG) was observed in $20 \%$ of the steroid-based cohort and in $29 \%$ of the steroid-free cohort. Overall, these results argue that sustained remission without corticosteroids is possible in anti-HMGCR myopathy.

Increasing the use of various SSI combinations in induction for refractory patients, but especially in maintenance strategies, may ultimately allow successful steroid-free and IVIG-free maintenance therapy. The successful strategies used in refractory and relapsing patients were either to switch to another SSI or to add an additional SSI through a step-up strategy.

A striking $40 \%$ of patients $(n=22 / 55)$ presented initially with persistent hyperCKemia and normal strength. Statin discontinuation led to $a \geq 50 \%$ drop in CK levels in $27-31 \%$ of patients, hinting to a natural history-based window of opportunity for successful early treatment. Although some patients were treated years later while they still had normal strength, it is noteworthy that proximal weakness ensued in many patients, with a median delay to treatment of 21.6 months. The nearineluctability of progressive myopathy in patients presenting with hyperCKemia is one argument for early treatment. Another convincing argument for early treatment would be if accrual damage in untreated disease would hasten the appearance of refractory disease. In untreated anti-HMGCR myopathy, regenerating muscle cells express high levels of HMGCR, sustaining and perhaps intensifying the autoimmune response with time, even after statins are discontinued [3].

Multivariate analysis revealed a crucial therapeutic finding, namely that delay in initiating treatment, even with hyperCKemia alone, decreases the probability of a successful SSI monotherapy maintenance. Indeed, refractory anti-HMGCR myopathy is frequent in patients with limb-girdle muscular dystrophy-like presentation [17], illustrating the consequence of delaying treatment. Moreover, early treatment might offer hope for drug-free remission, as achieved by 4 patients in the immediate treatment induction cohort. Weighting the advantages of safer, steroid-free induction against the consequences of delaying remission and missing a window of opportunity should be analyzed in future studies.

Limitations of the present study are a retrospective design, the lack of a standardized therapeutic approach, and the absence of documentation of corticosteroid toxicity. A strength of this study is the careful analysis of treatment strategies in 55 patients representing the full spectrum of statin-induced anti-HMGCR myopathy. Another strength is the long follow-up that allowed analysis of both induction and maintenance strategies, leading to the suggestion that early treatment with IVIG plus an SSI, with or without corticosteroids, is appropriate in 
most patients. Indubitably, randomized trials of IVIGbased initial treatment strategies in statin-induced antiHMGCR myopathy are needed, leading to individualized treatment tailored to disease severity.

\section{Conclusion}

In summary, the present study convincingly expanded the spectrum of anti-HMGCR myopathy to include isolated hyperCKemia, demonstrated the efficacy of steroid-free induction strategies in selected patients, validated the proposed Triple steroid/IVIG/SSI induction strategy, and confirmed that steroid-free maintenance is an achievable goal, occasionally through the use of SSI combinations or an SSI/IVIG maintenance. Finally, avoiding delays in treatment, most notably in patients with normal strength, may reset the natural history of anti-HMGCR myopathy from a refractory entity to a treatable disease.

\section{Supplementary information}

Supplementary information accompanies this paper at https://doi.org/10. 1186/s13075-019-2093-6.

Additional file 1 : Table S1. Definitions for therapy, remission, maintenance and severity of anti-HMGCR myopathy.

Additional file $\mathbf{2}$ : Table S2. Identifying therapeutic subgroups within the STATIN-PHESEMO study.

Additional file 3 : Table S3. Chronology of events leading to corticosteroid-free treatment of patients with anti-HMGCR myopathy $(N=14)$.

Additional file 4 : Table S4. Corticosteroid-free induction and maintenance therapy of patients with anti-HMGCR myopathy $(N=14)$

Additional file 5 : Table S5. Chronology of events, from disease onset to maintenance therapy, in patients with anti-HMGCR myopathy presenting with normal strength $(N=22)$

\section{Abbreviations \\ ABA: Abatacept; ALLO: Allopurinol; AZA: Azathioprine; CK: Creatine kinase; ENMC: European Neuromuscular Centre; HMGCR: 3-Hydroxy-3-methyl- glutaryl-coenzyme A reductase; IVIG: Intravenous immunoglobulins; MMF: Mycophenolate mofetil; MAC: Membrane attack complex; MRI: Magnetic resonance imaging; MTX: Methotrexate; RTX: Rituximab; SSI: Steroid-sparing immunosuppressant}

\section{Acknowledgements}

The authors thank Gemma Pérez, Meifeng Zhang, and Haiyan Hou for laboratory assistance.

\section{Authors' contributions}

$A M, Y T$, and JLS contributed to the design and conceptualization of the study. $Y T$ and JLS contributed to the study supervision and coordination. AM, YT, and $J \mathrm{LS}$ contributed to the analysis and interpretation of the data. $\mathrm{AM}, \mathrm{MH}$, and $\mathrm{SH}$ contributed to the statistical analysis. $A M, Y T$, and $J L S$ drafted the manuscript. YT, JD, GOL, OLC, SH, BH, JBT, AMM, SHu, VM, ER, JTG, SC, MH, JN, JPM, FZ, EV, JPR, ML, EKOF, JF, BE, JK, SL, RM, Cl, ID, VL, HC, INT, MJF, and JLS had major role in the data acquisition. YT, AM, JD, GOL, OLC, Sh, BH, JBT, AMM, Shu, VM, ER, JTG, SC, MH, JN, JPM, FZ, EV, JPR, ML, EKOF, JF, BE, JK, SL, RM, CI, ID, VL, HC, INT, $M J F$, and JLS revised the manuscript for intellectual content. JLS obtained the funding. All authors read and approved the final manuscript.

\section{Funding}

This work was supported in part by a grant from the Canadian Institutes of Health Research (MOP-142211 to JLS), by Department of Veterans Affairs Medical Research Funds (to INT), and by donations from Sclérodermie
Québec (JLS) and Mrs. Gisèle Sarrazin-Locas (JLS) in support of the Autoimmunity Research Laboratory, CHUM Research Center. JLS holds the University of Montreal Scleroderma Research Chair.

\section{Availability of data and materials}

Anonymized data not published within the article will be shared upon request from any qualified investigator.

\section{Ethics approval and consent to participate}

The STATIN-PHESEMO study was approved by the CHUM Research Ethics Committee (reference number 2015-5607-CE14.248) and by the Research Ethics Committees of Hôpital du Sacré-Coeur (2014-1042), Centre Hospitalier Affilié Universitaire Régional de Trois-Rivières (2014-028-03), and Hôpital Maisonneuve-Rosemont (2015-639-CER14107).

\section{Consent for publication}

All authors gave their consent for publication.

\section{Competing interests}

The authors declare that they have no competing interests.

\section{Author details}

${ }^{1}$ Faculté de médecine, Université de Strasbourg, Service de rhumatologie et Centre de références des maladies autoimmunes rares, Hôpitaux universitaires de Strasbourg, Strasbourg, France. ${ }^{2}$ Department of Medicine, Faculty of Medicine, University of Montreal, Montreal, Québec, Canada. ${ }^{3}$ Division of Rheumatology, Hôpital du Sacré-Coeur, Montreal, Québec, Canada. ${ }^{4}$ Division of Rheumatology, Centre hospitalier affilié universitaire régional de Trois-Rivières, Trois-Rivières, Québec, Canada. ${ }^{5}$ Division of Rheumatology, Centre Hospitalier de l'Université de Montréal (CHUM), 264, Boulevard René-Lévesque Est, Montréal, Québec H2X 1P1, Canada. ${ }^{6} \mathrm{CHUM}$ Research Center, Montréal, Québec, Canada. ${ }^{7}$ Service de médecine interne et immunologie clinique, Hôpital Pitié-Salpêtrière, Assistance publique Hôpitaux de Paris, Paris, France. ${ }^{8}$ Division of Internal Medicine, Hôpital du Sacré-Coeur, Montréal, Québec, Canada. ${ }^{9}$ Faculty of Pharmacy, Laval University, Québec City, Québec, Canada. ${ }^{10}$ Division of Rheumatology, Hôpital Maisonneuve-Rosemont, Montréal, Québec, Canada. ${ }^{11}$ Department of Medicine, McGill University, Montreal, Canada. ${ }^{12}$ Division of Rheumatology, Jewish General Hospital, Montreal, Canada. ${ }^{13}$ Lady Davis Institute, Jewish General Hospital, Montreal, Canada. ${ }^{14}$ Department of Neurology, McGill University, Montreal, Canada. ${ }^{15}$ Montreal Neurological Institute and Hospital, Montreal, Canada. ${ }^{16}$ Department of Pathology and Cell Biology, Faculty of Medicine, University of Montreal, Montreal, Canada. ${ }^{17}$ Department of Pathology, Hôpital Maisonneuve-Rosemont, Montreal, Canada. ${ }^{18}$ Department of Pathology, Hôpital Sainte-Justine, Montreal, Canada. ${ }^{19}$ Department of Pathology, McGill University, Montreal Neurological Institute and Hospital, Montreal, Québec, Canada. ${ }^{20}$ Department of Medicine, Sherbrooke University, Sherbrooke, Québec, Canada. ${ }^{21}$ Division of Neurology, Hôpital

Charles-Lemoyne, Longueuil, Canada. ${ }^{22}$ Division of Rheumatology, Hôpital Charles-Lemoyne, Longueuil, Québec, Canada. ${ }^{23} \mathrm{Hôpital}$ du Haut-Richelieu, Saint-Jean-sur-Richelieu, Québec, Canada. ${ }^{24}$ Departement of Medicine, Laval University, Québec City, Canada. ${ }^{25}$ Centre Hospitalier Universitaire de Québec, Québec City, Québec, Canada. ${ }^{26}$ Veterans Affairs Medical Center, University of Oklahoma Health Sciences Center, Oklahoma City, USA. ${ }^{27}$ Oklahoma Medical Research Foundation, Oklahoma City, OK, USA. ${ }^{28}$ Department of Medicine, Cumming School of Medicine, University of Calgary, Calgary, Alberta, Canada.

Received: 2 October 2019 Accepted: 23 December 2019

Published online: 08 January 2020

\section{References}

1. Needham M, Fabian V, Knezevic W, et al. Progressive myopathy with upregulation of $\mathrm{MHC}-\mathrm{I}$ associated with statin therapy. Neuromuscul Disord. 2007;17:194-200.

2. Christopher-Stine L, Casciola-Rosen LA, Hong G, et al. A novel autoantibody recognizing 200-kd and 100-kd proteins is associated with an immunemediated necrotizing myopathy. Arthritis Rheum. 2010;62:2757-66.

3. Mammen AL, Chung T, Christopher-Stine L, et al. Autoantibodies against 3hydroxy-3-methylglutaryl-coenzyme A reductase in patients with statinassociated autoimmune myopathy. Arthritis Rheum. 2011;63:713-21. 
4. Senécal JL, Raynauld JP, Troyanov Y. A new classification of autoimmune myositis. Arthritis Rheumatol. 2017;69:878-84.

5. Pinal-Fernandez I, Casal-Dominguez M, Carrino JA, et al. Thigh muscle MRI in immune-mediated necrotising myopathy: extensive oedema, early muscle damage and role of anti-SRP autoantibodies as a marker of severity. Ann Rheum Dis. 2017;76:681-7.

6. Chung T, Christopher-Stine L, Paik JJ, et al. The composition of cellular infiltrates in anti-HMG-CoA reductase-associated myopathy. Muscle Nerve. 2015;52:189-95.

7. Allenbach Y, Drouot L, Rigolet A, et al. Anti-HMGCR autoantibodies in European patients with autoimmune necrotizing myopathies: inconstant exposure to statin. Medicine (Baltimore). 2014:93:150-7.

8. Troyanov Y, Landon-Cardinal O, Fritzler MJ, et al. Atorvastatin-induced necrotizing autoimmune myositis: an emerging dominant entity in patients with autoimmune myositis presenting with a pure polymyositis phenotype. Medicine (Baltimore). 2017:96:e5694.

9. Grable-Esposito P, Katzberg HD, Greenberg SA, et al. Immune-mediated necrotizing myopathy associated with statins. Muscle Nerve. 2010;41:185-90.

10. Kassardjian CD, Lennon VA, Alfugham NB, et al. Clinical features and treatment outcomes of necrotizing autoimmune myopathy. JAMA Neurol. 2015;72:996-1003.

11. Tiniakou E, Pinal-Fernandez I, Lloyd TE, et al. More severe disease and slower recovery in younger patients with anti-3-hydroxy-3-methylglutary--coenzyme A reductaseassociated autoimmune myopathy. Rheumatology (Oxford). 2017;56:787-94.

12. Landon-Cardinal O, Allenbach Y, Soulages A, et al. Rituximab in the treatment of refractory anti-HMGCR immune-mediated necrotizing myopathy. J Rheumatol. 2018;46:623-27.

13. Arouche-Delaperche $L$, Allenbach $Y$, Amelin $D$, et al. Pathogenic role of anti-signal recognition protein and anti-3-Hydroxy-3-methylglutaryl-CoA reductase antibodies in necrotizing myopathies: myofiber atrophy and impairment of muscle regeneration in necrotizing autoimmune myopathies. Ann Neurol. 2017;81:538-48.

14. Allenbach $Y$, Arouche-Delaperche L, Preusse $C$, et al. Necrosis in anti-SRP(+) and anti-HMGCR(+)myopathies: role of autoantibodies and complement. Neurology. 2018;90:e507-e17.

15. Bergua C, Chiavelli H, Allenbach Y, et al. In vivo pathogenicity of lgG from patients with anti-SRP or anti-HMGCR autoantibodies in immune-mediated necrotising myopathy. Ann Rheum Dis. 2019;78:131-9.

16. Allenbach Y, Mammen AL, Benveniste O, et al. 224th ENMC Internationa Workshop: clinico-sero-pathological classification of immune-mediated necrotizing myopathies. Zandvoort, The Netherlands, 14-16 October 2016. Neuromuscul Disord. 2018:28:87-99.

17. Mohassel P, Landon-Cardinal O, Foley AR, et al. Anti-HMGCR myopathy may resemble limb-girdle muscular dystrophy. Neurol Neuroimmunol Neuroinflamm. 2019;6:523.

18. Mammen AL, Tiniakou E. Intravenous immune globulin for statin-triggered autoimmune myopathy. N Engl J Med. 2015;373:1680-2.

19. Basharat P, Lahouti AH, Paik JJ, et al. Statin-induced anti-HMGCR-associated myopathy. J Am Coll Cardiol. 2016;68:234-5.

20. Nzeusseu A, Brion F, Lefebvre C, et al. Functional outcome of myositis patients: can a low-dose glucocorticoid regimen achieve good functional results? Clin Exp Rheumatol. 1999:17:441-6.

21. Miloslavsky EM, Naden RP, Bijlsma JW, et al. Development of a Glucocorticoid Toxicity Index (GTI) using multicriteria decision analysis. Ann Rheum Dis. 2017;76:543-6.

22. Mammen AL. Statin-associated autoimmune myopathy. N Engl J Med. 2016;374:664-9.

23. Meriggioli MN. The clinical spectrum of necrotizing autoimmune myopathy: a mixed bag with blurred lines. JAMA Neurol. 2015;72:977-9.

24. Dalakas MC. Necrotising autoimmune myopathy (NAM): antibodies seem to be specific markers in aiding diagnosis. J Neurol Neurosurg Psychiatry. 2016;87:1037.

25. Smolen JS, Aletaha D, Bijlsma JW, et al. Treating rheumatoid arthritis to target: recommendations of an international task force. Ann Rheum Dis. 2010;69:631-7.

26. Oddis CV. Update on the pharmacological treatment of adult myositis. Intern Med. 2016;280:63-74.

27. Oddis CV, Medsger TA Jr. Relationship between serum creatine kinase level and corticosteroid therapy in polymyositis-dermatomyositis. J Rheumatol. 1988;15:807-11.

28. Werner JL, Christopher-Stine L, Ghazarian SR, et al. Antibody levels correlate with creatine kinase levels and strength in anti-3-hydroxy-3-methylglutarylcoenzyme A reductase-associated autoimmune myopathy. Arthritis Rheum. 2012;64:4087-93.

\section{Publisher's Note}

Springer Nature remains neutral with regard to jurisdictional claims in published maps and institutional affiliations.

\section{Ready to submit your research? Choose BMC and benefit from}

- fast, convenient online submission

- thorough peer review by experienced researchers in your field

- rapid publication on acceptance

- support for research data, including large and complex data types

- gold Open Access which fosters wider collaboration and increased citations

- maximum visibility for your research: over $100 \mathrm{M}$ website views per year

At BMC, research is always in progress.

Learn more biomedcentral.com/submissions 